\title{
ENSAMBLAJES DE ANUROS EN TRES ZONAS CON INTERVENCIÓN HUMANA EN EL PARQUE NACIONAL NATURAL LOS KATÍOS (COLOMBIA)*
}

\author{
Carlos E. Burbano-Yandi', Wilmar Bolivar-García ${ }^{2}$, Alan Giraldo
}

\section{Resumen}

Se evaluó la estructura y composición de los ensamblajes de anuros en tres zonas con diferente grado de intervención humana en el PNN Los Katíos, las cuales fueron el bosque El Tendal (bosque sucesión tardía), un bosque de Teca (bosque transformado) y el Poblado Sautatá (bosque urbanizado). Se evaluó la representatividad del muestreo utilizando el estimador no paramétrico Jackknife 1, y se describió el patrón de diversidad utilizando los índices de riqueza de especie, dominancia de Simpson (Ds), equitabilidad de Pielou (J) e incertidumbre de Shannon-Wiener $(\mathrm{H})$. Se utilizó la técnica de re-muestreo (bootstrapping) para comparar los atributos de diversidad establecidos, y se evaluó la similitud entre los ensamblajes mediante un análisis de agrupamiento pareado no ponderado (UPMGA) utilizando el índice de similitud de Bray-Curtis. Se registraron 507 individuos pertenecientes a 20 especies, incluidas en nueve familias y 14 géneros. Con el esfuerzo de muestreo realizado se registró el $68 \%$ de especies esperadas en el Poblado Sautatá, el 72\% en el bosque de Teca y el $87 \%$ en el bosque El Tendal. La mayor riqueza, menor dominancia, mayor equitabilidad y mayor diversidad de anfibios se registró en el bosque El Tendal (13 especies; Ds =0,22; J =0,75; H'=1,91 bits ind ${ }^{-1}$ ), seguida por el bosque de Teca (10 especies; Ds $=0,22 ; \mathrm{J}=0,76 ; \mathrm{H}^{\prime}=1,75$ bits ind $\mathrm{d}^{-1}$, siendo mayor la dominancia y significativamente menor la diversidad en el Poblado Sautatá (10 especies;Ds $=0,44 ; \mathrm{J}=0,55 ; \mathrm{H}^{\prime}=1,26$ bits ind ${ }^{-1}$ ). Rhaebo haematiticus, Rhaebo hypomelas, Espadarana callistomma, Hypsiboas rosenbergì Lithobates vaillanti solo fueron capturadas en el bosque El Tendal, mientras que Smilis casordida solo fue capturada en el bosque de Teca, y Rhinella marina, Scinax boulengeri y Leptodactylus insularum en el Poblado Sautatá. La mayor diversidad y el mayor número de especies exclusivas que fueron registradas en el bosque El Tendal probablemente estén relacionados con una mayor heterogeneidad de hábitat, lo que proporciona una mayor oferta de recursos y protección contra la desecación.

Palabras clave: anuros, Bosque húmedo tropical, bosque en regeneración, Chocó biogeográfico, diversidad, factores bióticos, hábitats modificados.

\footnotetext{
*FR: 2-IX-2014. FA: 12-III-2015

${ }^{1}$ Biólogo. Facultad de Ciencias Naturales y Exactas, Departamento de Biología, Grupo de Investigación en Ecología Animal. Universidad del Valle. Cali, Colombia. E-mail: burbanoyandi.c.e@gmail.com

${ }^{2}$ Doctor. Facultad de Ciencias Naturales y Exactas, Departamento de Biología, Grupo de Investigación en Ecología Animal. Universidad del Valle. Cali, Colombia. E-mail: wilmar.bolivar@correounivalle.edu.co

${ }^{3}$ Doctor. Facultad de Ciencias Naturales y Exactas, Departamento de Biología, Grupo de Investigación en Ecología Animal. Universidad del Valle. Cali, Colombia. E-mail:alan.giraldo@correounivalle.edu.co
} 


\title{
ANURA ASSEMBLAGES IN THREE AREAS WITH HUMAN INTERVENTION IN THE NATIONAL NATURAL PARK LOS KATÍOS (COLOMBIA)
}

\begin{abstract}
The structure and composition of anuran assemblages were evaluated in three zones with differing degree of human intervention at Los KatíosNational Natural Park. The zones were the forest of El Tendal (late successional forest), a forest of teak (intervened forest) and the village of Sautatá (urbanized forest). Sample representativeness was evaluated using the non-parametric estimator Jackknife 1. The diversity pattern was described using species richness indices, Simpson's dominance (Ds), Pielou's equitability $(\mathrm{J})$ and Shannon-Wiener $(\mathrm{H})$ uncertainty index. The assemblage attributes were compared using bootstrapping techniques and similitude among assemblages was evaluated withan UPMGA hierarchical clustering method using the Bray-Curtis index. A total of 507 individuals belonging to 20 species were recorded, including nine families and 14 genera. The sampling effort yielded $68 \%$ of expected species in the village of Sautatá, $72 \%$ of expected species in the teak plantation and 87\% of expected species in El Tendal forest. The highest richness, lowest dominance, highest equitability and highest amphibian diversity were recorded at El Tendal forest (13 species; Ds $=0.22 ; \mathrm{J}=0.75 ; \mathrm{H}^{\prime}=1.91$ bits ind1 ), followed by the teak forest ( 10 species; Ds $=0.22 ; \mathrm{J}=0.76 ; \mathrm{H}^{\prime}=1.75$ bits ind- 1 ). Dominance was higher and diversity was significantly lower at the village of Sautatá (10 species; Ds $=0.44 ; \mathrm{J}=0.55 ; \mathrm{H}^{\prime}=1.26$ bits ind-1). Rhaebo haematiticus, Rhaebo hypomelas, Espadarana callistomma, Hypsiboas rosenbergi and Lithobates vaillanti were captured only at the Tendal forest, Smilis casordida was only captured at the teak forest, and Rhinella marina,Scinax boulengeri and Leptodactylus insularum were only captured at the village of Sautatá. The highest diversity and highest number of exclusive species were recorded at the El Tendal forest. This result is probably related to a higher habitat heterogeneity, which would provide a higher number of refuges and protection against desiccation for anurans.
\end{abstract}

Key words: Chocó, diversity, frogs,tropical rain forest, recovery forest, altered habitat.

\section{INTRODUCCIÓN}

En Colombiase encuentra una gran diversidad de anfibios, con alrededor de 781 especies descritas hasta este momento (FROST, 2014). Paradójica y lamentablemente, también posee un alto número de especies en peligro,existiendo 275 especies catalogadas bajo distintas categorías de amenaza, siendo los anuros (ranas y sapos) los más amenazados (IUCN, 2014). En general, se ha reconocido a la fragmentación, la modificación y el deterioro de los hábitats naturales como los principales factores que atentan contra la supervivencia de estas especies (RUEDA-ALMONACID et al., 2004;WAKE \&VREDENBURG, 2008; CASTRO-H.\& BOLÍVAR-G., 2010; HEATWOLE \& WILKINSON, 2012; CAMPOS et al.,2013). En este sentido, se ha demostrado que cambios en la estructura de la vegetación delos bosques pueden actuar sobre los ensamblajes de anfibios, al producirse la pérdida directa de hábitats, con la 
consecuente reducción de microhábitats disponibles, pérdida de nichos tróficos y cambios microclimáticos, entre otros (GRANT et al., 1994; HERRERA-M.et al., 2004; URBINA-C.et al., 2006; BECKER et al.,2007).

En ambientes tropicales los anuros responden a los cambios ambientales y de estructura vegetal de distintas maneras, evidenciándose dichos cambios en la riqueza y composición de especies (VARGAS \& BOLAÑOS, 1999; CÁCERES-A. \& URBINA-C., 2009). De forma general, se observa que cada especie de anfibio se distribuye en su entorno de acuerdo a los microhábitats que se encuentran disponibles, demostrando cierto grado de tolerancia en algunos ambientes modificados (URBINA-C. \& LONDOÑO-M., 2003; GARCÍA et al., 2005a).

El Parque Nacional Natural (PNN) Los Katíos, hace parte del Sistema Nacional de Áreas Protegidas (SINAP) y se encuentra ubicado al noroccidente de Colombia en la región del Darién y Urabá, en jurisdicción de los departamentos de Chocó y Antioquia. Esta área contribuye a la protección de las zonas de vida de bosque húmedo tropical (bh-T) y el bosque muy húmedo tropical (bmh-T), encontrándose representados ecosistemas de bosques inundables en llanura aluvial, ciénagas, bosques riparios en llanura aluvial, selva tropical en serranía aislada,entre otros(MORENO-VALDERRAMA et al.,2006). De acuerdo con el plan de manejo de esta área protegida, se estima que el PNN Los Katíos alberga cerca de 550 especies de vertebrados terrestres, siendo los anuros uno de los grupos taxonómicos menos evaluados dentro del parque (MORENO-VALDERRAMA et al.,2006), aunque los bosques de tierras bajas delChocó biogeográfico han sido identificados como una de las zonas biogeográficas de Colombia con mayor diversidad de anfibios (LYNCH et al.,1997).

Ha sido ampliamente documentado que las variaciones temporales en la abundancia de anuros pueden convertirse en una herramienta de alerta temprana ante los cambios estructurales de un hábitat (STORFER, 2003; MONTES et al., 2004; GARCÍA-R. et al., 2005b; 2006; ABARCA, 2012). Por lo tanto, conocer los atributos ecológicos de los ensamblajes presentes en un área protegida, permitirá fortalecer las acciones de manejo y conservación implementadas, e incluso genera valores de referencia para evaluar la efectividad de estas acciones y permite evaluar cuantitativamente la integridad ecológica del sistema (REZA \& ABDULLAH, 2011; KEENELYSIDE et al.,2012). Con la presente investigación se contribuye al conocimiento de la fauna de anfibios presentes en algunas áreas accesibles del PNN Los Katíos, y se describen algunos atributos ecológicos de los ensamblajes de anuros en tres zonas que presentan diferentes tipos de intervención antrópica.

\section{MATERIALES Y MÉTODOS}

\section{Área de estudio}

Para el desarrollo de esta investigación se seleccionaron tres zonas accesibles del PNN Los Katíos, que difieren en el grado de intervención humana al que están sometidas: el Poblado Sautatá, el bosque de Teca, y el bosque El Tendal (Figura1). En términos generales, la temperatura media anual en esta área es de $26,4^{\circ} \mathrm{C}$, con humedad relativa media anual del $85,2 \%$ y un régimen de lluvias monomodal, con un período de baja precipitación en los meses de diciembre a marzo, y un período de mayor precipitación y más húmedo entre los meses de mayo a noviembre (MORENO-VALDERRAMA et al., 2006). 


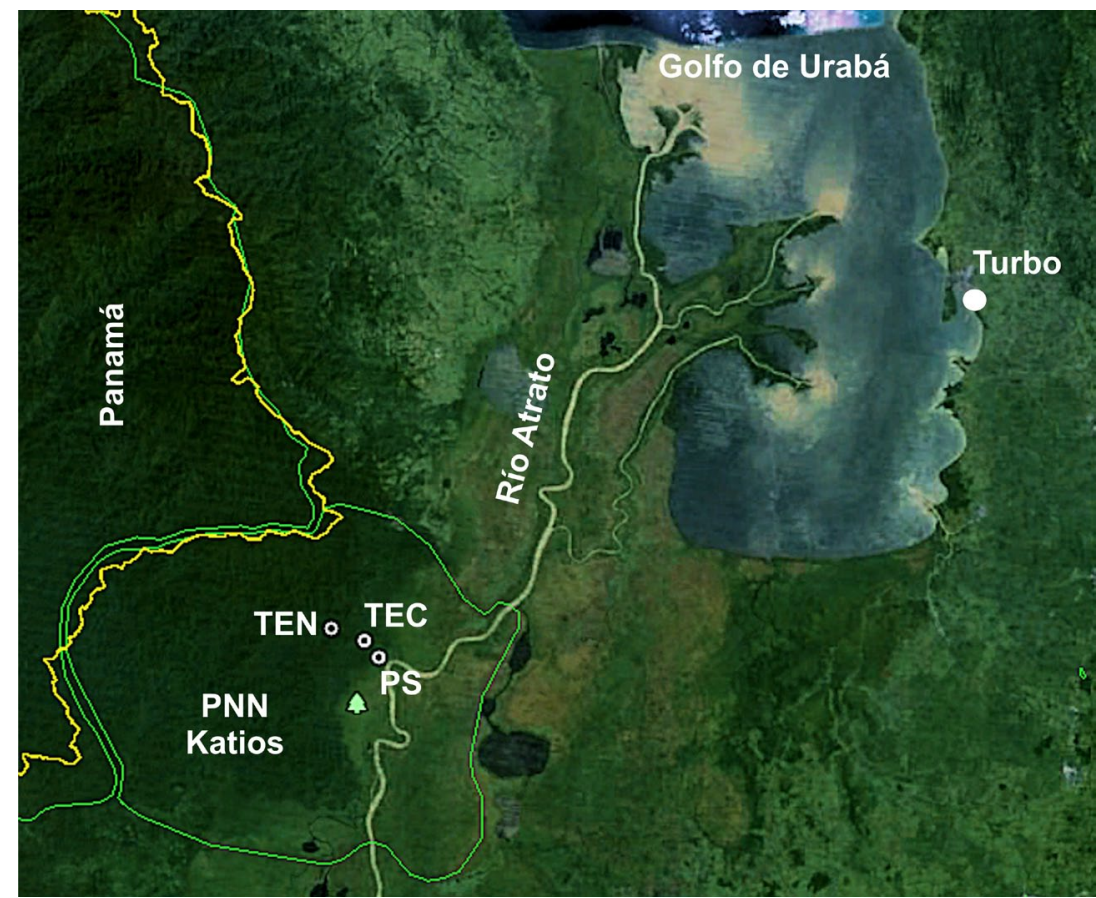

PS: Poblado Sautatá. TEC: bosque Teca. TEN: bosque El Tendal.

Fuente: Google Earth ${ }^{\circledR}$ imagen Landsat.

Figura 1. Ubicación de las zonas de estudio en el Parque Nacional Natural Los Katíos.

El Poblado Sautatá (PS) (7050'11,9” N - 77007'57,3”'W) fue la zona que presentó mayor intervención humana. Esta localidad históricamente ha sido el epicentro de las actividades agropecuarias que se han desarrollado en la región y en donde está ubicado el mayor asentamiento humano en el área protegida. La vegetación circundante se caracterizó por rastrojos altos en sucesión natural, árboles de poco diámetro y frutales como mango, ciruela y limón, en medio de amplias zonas de pastos junto a los que se desarrollaban bejucos espinosos asociados a melastomatáceas, rubiáceas y piperáceas, con la formación constante de charcos temporales debido a la presencia de animales como caballos y mulas.

El bosque de Teca (TEC) $\left(7^{\circ} 50^{\prime} 3,0^{\prime \prime} \mathrm{N}-77^{\circ} 08^{\prime} 32,7^{\prime \prime} \mathrm{W}\right)$ fue seleccionado e identificado como la zona de intervención media. Este sector está conformado por rodales de Tectona grandis inmersos en una matriz de rastrojo alto, con helechos y herbáceas en los bordes de los senderos. Aunque es un sector que ha sido destinado para su recuperación natural desde principios de los años ochenta, la presencia de los rodales de Teca ha limitado este proceso, registrándose principalmente especies vegetales características de procesos de sucesión secundaria como Cochlospermum sp., Piper sp., Canna spp., Hura crepitans, Enterolobium sp., Senna reticulata, Cecropia burriada, Inga spp., Spondias mombin, Ficus glabrata, Guazuma ulmifolia, Heliconia sp., Trema micrantha (MORENO-VALDERRAMA et al., 2006). 
El bosque El Tendal (TEN) (7051'23,5” N- 7709'55,2” W), se desarrolla a lo largo de la microcuenca de la quebrada El Tendal, y hasta antes de la declaratoria del área protegida estuvo afectado por entresaca de madera, razón por la cual fue considerado como la zona de intervención baja. La matriz de bosque está conformada por árboles de mediano porte de Trema micrantha, Cecropia arachnoidea, C. occidentalis, $C$. peltata, Ochroma lagopus y Heliocarpus popayanensis, con presencia de claros dominados por Miconia lepidota, M. nervosa, M. centrodesma, Psychotria uliginosa, P. marginata y P. deflexa (MORENO-VALDERRAMA et al., 2006). El estrato arbustivo está dominado por rastrojos altos de Chromolaena barranquillensis, Vernonia baccharoides, Piper marginatum, T.micrantha, H.popayanensis y Acalypha diversifolia, y la presencia de árboles y arbustos de sucesión avanzada de Ochroma pyramidale, Cecropia spp., T. micrantha, Spondias mombin, Heliconia mariae, $H$. latispatha, y algunos bejucos (MORENO-VALDERRAMA et al., 2006).

\section{Método de muestreo}

Se realizaron dos jornadas de muestreo, una en noviembre de 2011 (época húmeda) y otra en marzo de 2012 (época seca), cada una con duración de 20 días, con el propósito de abarcar los dos períodos climáticos descritos para la región. En cada zona se establecieron 20 trayectos lineales de $300 \mathrm{~m}$ de longitud y se utilizó la técnica de relevamiento por encuentro visual (REV) (HEYER et al., 1994) para realizar el censo de anuros, recorriendo 10 trayectos durante el día y 10 trayectos durante la noche. La búsqueda fue realizada por dos observadores entrenados, estableciendo 90 min como el tiempo efectivo de búsqueda en cada recorrido. Se definió una amplitud de banda efectiva de $5 \mathrm{~m}$ y una altura máxima de búsqueda de $3 \mathrm{~m}$. Cada individuo detectado fue identificado siguiendo la propuesta de FROST (2014) y se estableció el microhábitat ocupado al momento del avistamiento por cada individuo considerando las categorías propuestas por HEYER et al. (1994): hojarasca, hoja, rama, tronco caído, suelo desnudo, roca y tallo.

\section{Análisis de datos}

Se utilizó el estimador no paramétrico de riqueza de Jackknife 1, el cual considera el aporte de los registros únicos con relación al esfuerzo de muestreo, para establecer el número potencial de especies esperadas para cada zona (HELTSHE \& FORRESTER, 1983; GOTELLI \& COLWELL, 2011). A partir de este valor se determinó el porcentaje de representatividad del estudio (COLWELL \& CODDINGTON, 1995; INGARAMO et al., 2012; COLWELL, 2013). Para describir los atributos de cada ensamblaje, se utilizó el número de especies registradas y los índices de dominancia de Simpson (Ds), equidad Pielou (J) e incertidumbre de Shannon-Weiner $(\mathrm{H})$. Se realizó una comparación entre los atributos establecidos para cada ensamblaje utilizando la técnica de re-muestreo (bootstrapping) a partir de 1000 repeticiones aleatorias, y se evaluó la similitud entre los ensamblajes en relación a su composición de especies mediante un análisis de agrupamiento pareado no ponderado (UPMGA) a partir de una matriz de similitud de Bray-Curtis. Se utilizó el coeficiente de correlación cofenético $\left(r_{c}\right)$ para establecer la significancia del agrupamiento generado. Todos los análisis numéricos se realizaron utilizando el programa estadístico Past v.3. (HAMER et al., 2001; HAMER et al., 2005). Adicionalmente, se utilizaron los registros de las cinco especies más abundantes para evaluar su afinidad a un sustrato en particular utilizando una prueba de bondad de ajuste (Chi-cuadrado). 


\section{RESULTADOS}

El esfuerzo de muestreo en cada zona fue equivalente a 30 horas/hombre para un esfuerzo total de muestreo de 90 horas/hombre. Se registraron un total de 507 individuos durante todas las jornadas de muestreo, pertenecientes a 20 especies de anuros, distribuidas en 14 géneros y 7 familias (Figura 2). La familia con mayor representatividad fue Hylidae (seis especies), aunque la especie con mayor número de registros fue Crauga storraniformis de la familia Craugastoridae (Tabla 1).

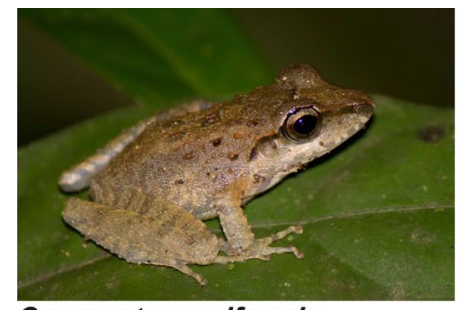

Craugastor raniformis

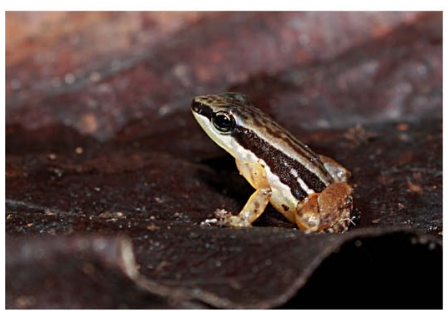

Colostethus pratti

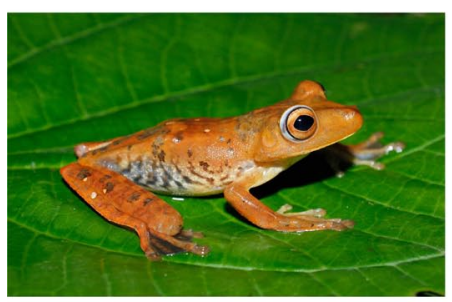

Hypsiboas boans

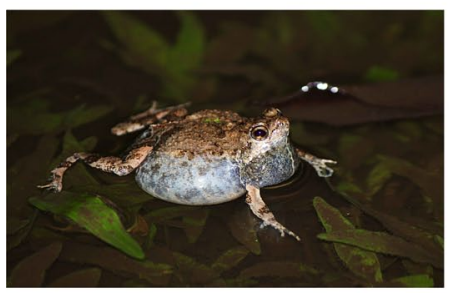

Engystomops pustulosus

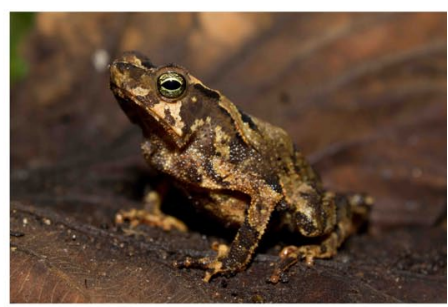

Rhaebo haematiticus

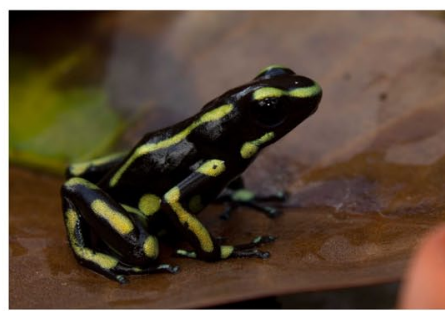

Dendrobates truncatus

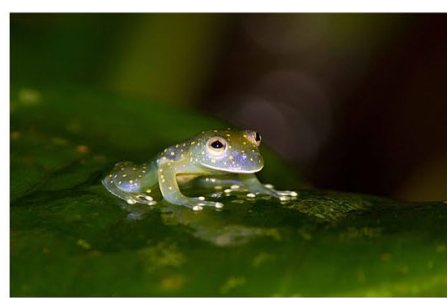

Cochranella euknemos

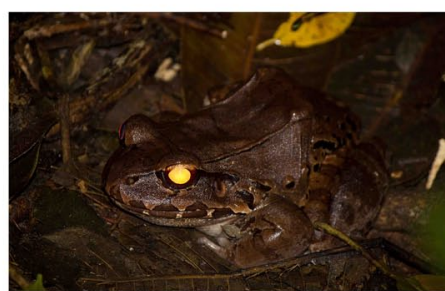

Leptodactylus pentadactylus

Fotografías: Diego Gómez ${ }^{\circledR}$.

Figura 2. Registro fotográfico de algunas de las especies de anfibios capturadas en el PNN Los Katíos durante las campańas de muestreo. 
Con base en el estimador de riqueza Jackknife 1, se estableció que el esfuerzo de muestreo permitió registrar entre el $68 \%$ al $87 \%$ de las especies esperadas para los sitios de estudio ( $\mathrm{PS}=68 \%, \mathrm{TEC}=72 \%, \mathrm{TEN}=87 \%$ ). En referencia a las especies exclusivas para cada sitio, en el bosque El Tendal se registró el mayor número con 5 especies, seguida del Poblado Sautatá con 2 y por último el bosque de Teca con una (Smilisca sordida) (Figura 3). El grado de similitud entre los ensamblajes de anuros descritos para el bosque El Tendal y el bosque de Teca fue del 59\%, mientras que el grado de similitud entre estos ensamblajes y el ensamble de anuros descrito para el Poblado Suatatá fue menor al 27\% (Figura 4), encontrando además que el ensamblaje de anuros del bosque El Tendal fue el de mayor riqueza específica, menor dominancia, mayor equitabilidad y mayor diversidad ( 13 especies; Ds=0,22; $\mathrm{J}=0,75 ; \mathrm{H}^{\prime}=1,91$ bits ind $^{-1}$.

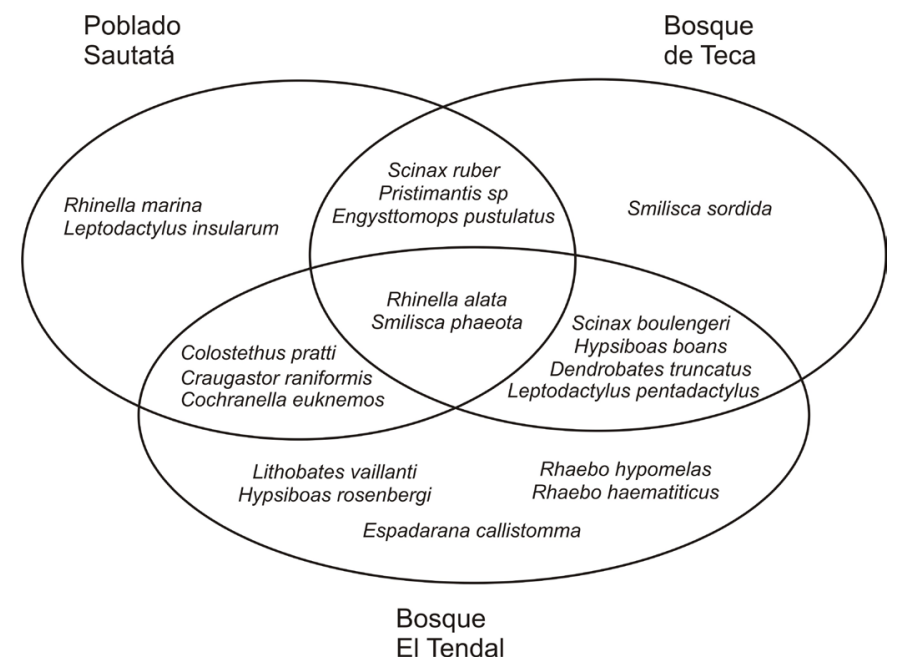

Figura 3. Composición taxonómica de los ensamblajes de anuros presentes en tres zonas con diferente grado de intervención humana en el PNN Los Katíos. Las áreas de traslape contienen especies compartidas entre zonas.

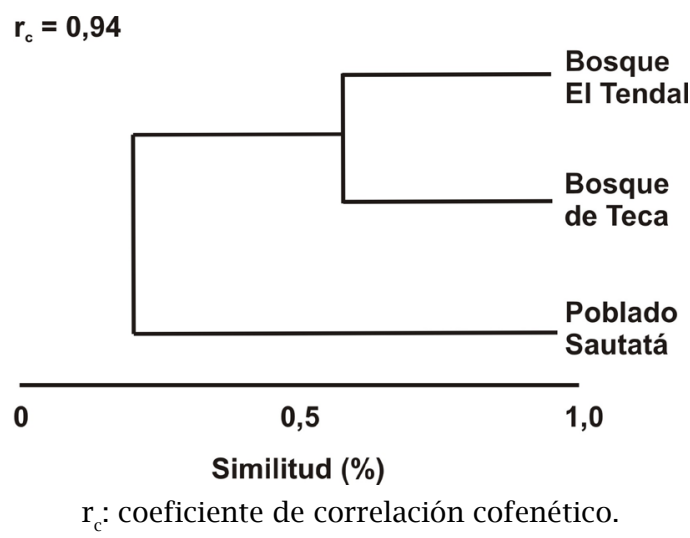

Figura 4. Dendrograma de similitud según el coeficiente de Bray-Curtis para las tres zonas con diferente grado de intervención humana en el PNN Los Katíos. 
No se detectaron diferencias significativas entre los atributos establecidos parael ensamblaje de anuros del bosque El Tendal y el bosque de Teca (10 especies; $\mathrm{Ds}=0,22, \mathrm{p}=0,92 ; \mathrm{J}=0,76, \mathrm{p}=0,43 ; \mathrm{H}^{\prime}=1,75$ bits ind $\left.^{-1}, \mathrm{p}=0,19\right)$. Aunque la riqueza específica del ensamblaje de anuros del Poblado Sautatá (10 especies) fue similar a la registrada en el bosque de Teca, este ensamblaje exhibió una mayor dominancia (Ds=0,44, $\mathrm{p}=0,0001)$, menor equidad $(\mathrm{J}=0,55, \mathrm{p}=0,0001)$, y menor diversidad $\left(\mathrm{H}^{\prime}=1,26\right.$ bits ind $\left.{ }^{-1}, \mathrm{p}<0,0002\right)$ que los ensamblajes de anuros del bosque de Teca $y$ el bosque El Tendal.

Las cinco especies utilizadas para realizar el análisis de afinidad de microhábitat exhibieron diferencias significativas en los patrones de uso aparente (Tabla 2). Craugastor raniformis, Rhinella alata y Engystomops pustulosus estuvieron asociadas preferentemente a las hojarasca; Colostethus pratti estuvo asociada preferentemente a zonas rocosas, mientras que Hypsiboas boans estuvo asociada preferentemente con las hojas.

Tabla 1. Listado de las especies registradas en las tres zonas intervenidas en el PNN Los Katíos en noviembre de 2011 y marzo de 2012.

\begin{tabular}{|c|c|c|c|c|c|}
\hline Familia & Especie & PS & TEC & TEN & Total \\
\hline \multirow[t]{4}{*}{ Bufonidae } & Rhaebo haematiticus & & & 15 & 15 \\
\hline & Rhaebo hypomelas & & & 1 & 1 \\
\hline & Rhinella alata & 15 & 33 & 36 & 84 \\
\hline & Rhinella marina & 9 & & & 9 \\
\hline \multirow[t]{2}{*}{ Centrolenidae } & Espadarana callistomma & & & 1 & 1 \\
\hline & Cochranella euknemos & & 2 & 3 & 5 \\
\hline \multirow[t]{2}{*}{ Craugastoridae } & Crauga storraniformis & & 57 & 96 & 153 \\
\hline & Pristimantis sp. & 1 & 1 & & 2 \\
\hline \multirow[t]{2}{*}{ Dendrobatidae } & Colostethus pratti & & 24 & 15 & 39 \\
\hline & Dendrobates truncatus & 1 & & 11 & 12 \\
\hline \multirow[t]{6}{*}{ Hylidae } & Hypsiboas boans & 1 & & 26 & 27 \\
\hline & Hypsiboas rosenbergi & & & 6 & 6 \\
\hline & Scinax boulengeri & 1 & & & 1 \\
\hline & Scinax ruber & 3 & 1 & & 4 \\
\hline & Smilisca sordida & & 11 & & 11 \\
\hline & Smilisca phaeota & 1 & 3 & 2 & 6 \\
\hline \multirow[t]{3}{*}{ Leptodactylidae } & Engystomops pustulosus & 74 & 19 & & 93 \\
\hline & Leptodactylus insularum & 10 & & & 10 \\
\hline & Leptodactylus pentadactylus & & 7 & 12 & 19 \\
\hline Ranidae & Lithobates vaillanti & & & 9 & 9 \\
\hline Total de registros & & 116 & 158 & 233 & 507 \\
\hline Riqueza de especies & & 10 & 10 & 13 & \\
\hline Riqueza de especies exclusivas & & 2 & 1 & 5 & \\
\hline
\end{tabular}

PS: Poblado Sautatá. TEC: bosque de Teca. TEN: bosque El Tendal. 
Tabla 2. Porcentaje de uso evidente del microhábitat por parte de cinco especies de anuros en zonas con diferente grado de intervención humana en el PNN Los Katíos.

\begin{tabular}{lccccccccc}
\hline Especie & Zona & HO & HJ & RA & TC & SD & RO & $\mathbf{c}^{\mathbf{2}}$ & p \\
\hline Rhinella alata & PS, TEC, TEN & 95 & 1 & 0 & 1 & 0 & 3 & 37,71 & 0,0001 \\
Crangastor raniformis & TEC, TEN & 67 & 17 & 3 & 5 & 2 & 6 & 10,16 & 0,0170 \\
Colostethus pratti & TEC, TEN & 26 & 0 & 0 & 5 & 0 & 69 & 6,00 & 0,0500 \\
Hypsiboas boans & PS, TEN & 0 & 86 & 11 & 4 & 0 & 0 & 58,03 & 0,0001 \\
Engystomopspustulosus & PS, TEC & 100 & 0 & 0 & 0 & 0 & 0 & 31,49 & 0,0001 \\
\hline
\end{tabular}

HO: hojarasca. HJ: hojas. RA: ramas. TC: tronco caído. SD: suelo desnudo. RO: rocas. PS: Poblado Sautatá, TEC: bosque Teca. TEN: bosque El Tendal.

\section{DISCUSIÓN}

La riqueza de especies registradas durante esta investigación en el Poblado Sautatá, el bosque de Teca y el bosque El Tendal, representan el 60\% de las especies que han sido registradas para la zona del PNN Los Katíos (LYNCH \& SUÁREZ, 2004). Además, se adicionan a este listado el registro formal de Rhaebo hypomelas, Espadarana callistomma, Cochranella euknemos, Hypsiboas rosenbergi, Smilisca sordida, Smilisca phaeota y Leptodactylus pentadactylus, lo que incrementa a 40 las especies de anuros registradas en esta área protegida.

La especie más abundante en la zona de estudio fue Craugas torraniformis, correspondiendo la mayoría de los registros a individuos juveniles durante los censos diurnos. Esta tendencia podría ser una estrategia para reducir la competencia con individuos adultos de esta especie, los cuales generalmente son de hábitos nocturnos (LYNCH \& MYERS, 1983; VARGAS \& CASTRO-H., 1999). En términos generales, el ensamblaje de anuros en las zonas de estudio estuvo dominado por especies de la familia Hylidae. Estas especies son frecuentemente reportadas como tolerantes a las condiciones que ofrecen los ambientes intervenidos, ya que exhiben una alta variedad de modos reproductivos, pueden fácilmente explotar los recursos de los hábitats artificiales, y la presencia de discos adhesivos en sus dedos les permiten fácilmente ocupar el estrato vertical del hábitat (DUELLMAN, 1990; HADDAD \& SAWAYA, 2000).

Por el contrario, especies como Dendrobates truncatus y E. callistomma presentan limitaciones para poder permanecer en ambientes intervenidos. Específicamente, D.truncatus requiere condiciones particulares de humedad a nivel de la hojarasca para depositar los huevos, y fuentes de agua cercanas para el desarrollo de los renacuajos (GUAYARAN-BARRAGÁN \& BERNAL, 2012), mientras que E.callistomma necesita de quebradas con flujo de agua permanente para su reproducción, $y$ la presencia de vegetación suspendida para poder adherir su masa de huevos (GUAYASAMÍN \& TRUEB, 2007; GUAYASAMÍN et al., 2009). Considerando estas limitaciones, la presencia de estas especies sería poco probable en ambientes sin cuerpos de agua permanentes o con poca vegetación, tal como ocurre en el Poblado Sautatá. 
En ambientes intervenidos que todavía mantienen parte de su estructura espacial inicial, como el bosque de Teca o el bosque El Tendal, la cercanía a bosques secundarios en recuperación puede llegar a modular la estructura y composición del ensamblaje de anuros presentes. Cuando se desarrolla el proceso de recuperación de un hábitat que ha sido intervenido, y este mismo se encuentra adyacente a un bosque secundario, la probabilidad de desplazamiento de especies tolerantes desde el bosque hacia la zona de recuperación es mayor, llegando incluso a convertirse la zona de recuperación en una zona de tránsito de especies, condición que podría explicar el grado de similitud entre los ensamblajes de las áreas evaluadas (DE SOUZA et al., 2008). No obstante, no se puede determinar la exclusividad de las especies por cierto tipo de área debido a que el método de muestreo utilizado hizo énfasis en la recolección en estratos bajos, y está influido por la imagen de búsqueda de cada investigador (URBINA-C. \& LONDOÑO-M., 2003).

Las características de hábitat asociadas al bosque El Tendal podrían estar generando condiciones microclimáticas menos variables a la vez que una mayor cantidad de microhábitats, que estarían siendo aprovechados por algunas especies de anuros como protección contra la desecación (OSORNO, 1999; HERRERA-M. et al., 2004; URBINA-C. et al., 2006).Esto podría explicar la mayor riqueza de especies incluyendo las exclusivas de esta área, como $R$. haematiticus, $R$. hypomelas, E. callistomma, $H$. rosembergi y L. vaillanti en esta zona.

Aunque las modificaciones del entorno generadas por las construcciones humanas, la presencia de animales de cría o el establecimiento de cultivos pueden generar numerosos microhábitats, estos tienden a ser efímeros en el tiempo, por lo que solo aquellas especies que exhiben una amplia tolerancia a condiciones ambientales variables podrán hacer uso de esta oferta (ERNST \& RÖDEL, 2005; DE SOUZA et al., 2008). En este sentido, el ensamblaje de anuros en el Poblado Sautatá estuvo dominado en número por E. pustulosus, ya que esta especie tiende a aprovechar la formación de charcos temporales en las zonas de pastos abiertos que rodean las cabañas para reproducirse (MARSH et al., 1999; obs.pers.).

En un ensamblaje, cada especie de anuro requiere cubrir sus necesidades particulares de alimento, refugio y sustrato (HUEY, 1991; XIMENEZ et al., 2012). Cuando se evalúa a pequeña escala la elección de uso de sustrato que realizan los individuos de una especie, considerando las características físicas asociadas, se está evaluando la afinidad de sustrato (PIPER, 2011). En los anuros, la afinidad de sustrato generalmente depende de las adaptaciones particulares de las especies. Algunas especies, como $R$. alata, que presenta piel tuberculada y gruesa que la protege de la desecación, pueden ser generalistas en la escogencia del sitio de percha o sitios reproductivos (DUELLMAN \& TRUEB, 1986; EVANS et al., 1996), sin embargo, la carencia de adaptaciones morfológicas para trepar como la presencia de almohadillas adherentes y extremidades delgadas y largas, le impiden utilizar sustratos arbóreos. En contraste, C. raniformis y E. pustulosus, especies carentes de adaptaciones para evitar la desecación, necesitan de ambientes con porcentajes altos de humedad y que además los proteja de la radiación solar durante el día, condiciones que encuentran en un microhábitat como la hojarasca.

Aunque Colostethus pratti presentó una alta afinidad con el sustrato rocoso, siempre fue capturada en hábitat de quebrada, el cual ofrece una alta variedad de refugios contra predadores y protección contra la desecación. Las especies del género 
Colostethus generalmente se encuentran restringidas a pocos microhábitats debido a estrés hídrico, e incluso en los pocos reservorios de agua que quedan pueden llegar a agregarse un gran número de individuos (NAVAS et al.,2013; JONGSMA et al., 2014). Son especies que pueden tener una alta actividad en las inmediaciones de las quebradas que frecuentan permanentemente o pueden pasar la mayor parte del día ocultas dentro de la vegetación más baja, donde hay una mayor humedad relativa que en zonas abiertas (DUELLMAN \& TRUEB, 1986).

Para Hypsiboas boans la afinidad de microhábitat estaría relacionada con la presencia de almohadillas adherentes y extremidades delgadas y largas las cuales le permiten explotar el sustrato hoja, en los que además encuentran numerosos artrópodos que son parte fundamental de su dieta (MUÑOZ-GUERRERO et al., 2007). Además, por perchar más alto los machos podrían incrementar el éxito de atracción de hembras ya que reducen el efecto de atenuación de la vegetación sobre su canto de advertencia, como ha sido sugerido por DE SÁ et al. (2014) en Hypsiboas albopunctatus y por WELLS \& SCHWARTZ (1982) para Hyalinobatrachium fleischmanni.

A pesar de la importancia que tiene el bosque húmedo y el bosque muy húmedo tropical del Chocó biogeográfico para la conservación de los anfibios, aún no es claro el efecto que ha tenido la alteración de hábitat sobre la diversidad de anuros en esta región. Aunque los atributos ecológicos de los ensamblajes de anuros del bosque El Tendal (intervención baja) y el bosque de Teca (intervención media) en el PNN Los Katíos no fueron estadísticamente diferentes, probablemente este resultado esté influenciado por la dominancia en número de una sola especie, C. raniformis. Es importante destacar que cuando el hábitat está alterado, los ensamblajes de anuros tienden a tener una baja riqueza y se identifica la presencia de una especie dominante (FICETOLA \& DE BERNARDI, 2004). Esto hace necesario, que además de considerar la composición de los ensamblajes para definir acciones de manejo (PEARMAN, 1997; BECKER et al., 2010), también se tenga en cuenta el estado de conservación de las especies que los componen (JONGSMA et al., 2014; MENDOZA \& ARITA, 2014).

\section{AGRADECIMIENTOS}

A los funcionarios de Parques Nacionales de Colombia - PNN Los Katíos, por facilitar el apoyo logístico necesario para la realización de esta investigación, en especial a Valentina Zambrano, Nilson Mosquera, César Geller y Edgar Benítez. A Ximena García, Martín Llano Almario, Luisa Arcila, Diego Gómez y Ângela María González por su colaboración durante las jornadas de campo y laboratorio. Esta investigación fue parcialmente financiada por la Universidad del Valle, Parques Nacionales Naturales de Colombia y el Fondo Acción a través de la convocatoria realizada por el Fondo de Investigaciones Mono Hernández 2011, proyecto "Estrategia integral de conservación para tres especies de Atelopus amenazados de extinción en Parques Nacionales Naturales de Colombia”. Esta investigación hace parte del trabajo de grado desarrollado por uno de los autores, C.E.B.-Y., para obtener el grado académico de Biólogo, en la Universidad del Valle. 


\section{BIBLIOGRAFÍA}

ABARCA, J.G., 2012.- Cambios en la estructura de la comunidad de anuros (Amphibia: Anura) en el Cerro Chompipe, Costa Rica. Research Journal of the Costa Rican Distance Education University, 4(1): 9-15.

BECKER, C.G., FONSECA, C.R., HADDAD, C.F.B., BATISTA, R.F. \& PRADO, P.I., 2007.- Habitat split and the global decline of amphibians. Science, 318:1775-1777. DOI: 10.1126/science.1149374.

BECKER, C.G., LOYOLA, R.D., HADDAD, C.F.B. \& ZAMUDIO, K.R., 2010.- Integrating species life history traits and patterns of deforestation in amphibian conservation planning. Diversity and Distributions, 16(1), 10-19.: 10.1111/ j.1472-4642.2009.00625.x.

CÁCERES-A., S.P. \& URBINA-C., J.N., 2009.-Ensamblajes de anuros de sistemas productivos y bosques en el Piedemonte Llanero, Departamento del Meta, Colombia. Caldasia, 31(1): 175-194.

CAMPOS, F.S., BRITO, D. \& SOLÉ, M., 2013.-Threatened Amphibians and Their Conservation Status within the Protected Area Network in Northeastern Brazil. Journal of Herpetology, 47(2): 277-285. DOI: http:// dx.doi.org/10.1670/11-158.

CASTRO-H., F. \& BOLÍVAR-G., W., 2010.-Libro rojo de los anfibios del Valle del Cauca. Corporación Autónoma Regional del Valle del Cauca -CVC-, Cali, Colombia.

COLWELL, R.K., 2013.-EstimateS: Statistical estimation of species richness and shared species from samples. Version 9. Disponible en: http://viceroy.eeb.uconn.edu/EstimateS

COLWELL, R.K. \& CODDINGTON, J.A., 1995.-Estimating terrestrial biodiversity through extrapolation: 101-118 (en) HAWKSWORTH, D.L. (ed.) Biodiversity measurement and estimation. Chapman \& Hall, Nueva York, EEUU.

DE SÁ, F.P., ZINA, J. \& HADDAD, C.F.B., 2014.- Reproductive dynamics of the neotropical tree frog Hypsiboas albopunctatus (Anura, Hylidae). Journal of Herpetology, 48(2): 181-185. DOI: http://dx.doi. org/10.1670/12-193.

DE SOUZA, V.M., DE SOUZA, M.B. \& MORATO, E., 2008.-Efeitos da sucessão florestal sobre a anurofauna (Amphibia: Anura) da Reserva Catuaba e seu entorno, Acre, Amazônia sul-ocidental. Revista Brasileira de Zoologia, 25 (1): 49-57. DOI: http://dx.doi.org/10.1590/S0101-81752008000100008.

DUELLMAN, W.E., 1990.-Herpetofauna in Neotropical Rain Forest: Comparative Composition, History and Resource use: 455-505 (en) GENTRY, A.H. (ed.) Four Neotropical Rain Forest. Yale University Press, New Harven, USA.

DUELLMAN, W.E. \& TRUEB, L., 1986.-Biology of Amphibians. McGraw-Hill, Nueva York.

ERNST, R. \& RÖDEL, M.O., 2005.-Anthropogenically induced changes of predictability in tropical anuran assemblages. Ecology, 86(11): 3111-3118. DOI:http://dx.doi.org/10.1890/04-0800.

EVANS, M., YABER, C. \& HERO, J.M., 1996.- Factors influencing choice of breeding site byBufomarinus in its natural habitat. Copeia, 4: 904-912. DOI: 10.2307/1447653.

FICETOLA, G.F. \& DE BERNARDI, F., 2004.-Amphibians in a human-dominated landscape: the community structure is related to habitat features and isolation. Biological Conservation, 119: 219-230.DOI: 10.1016/j.biocon.2003.11.004.

FROST, D.R., 2014.- Amphibian Species of the World: an Online Reference. Version 6.0. American Museum of Natural History, New York. Electronic Database accessible at http://research.amnh.org/ herpetology/ amphibia/index.html

GARCÍA-R., J.C., CÁRDENAS-H., H. \& CASTRO-H., F., 2005a.-Relación entre la diversidad de anuros y los estados sucesionales de un Bosque muy húmedo Montano Bajo del Valle del Cauca, suroccidente colombiano. Caldasia, 29(2): 363-374.

GARCÍA-R., J.C., CASTRO-H., F., \& CÁRDENAS-H., H., 2005b.-Relación entre la distribución de anuros y variables del hábitat en el sector La Romelia del Parque Nacional Natural Munchique (Cauca, Colombia). Caldasia, 27(2): 299-310.

GOTELLI, N.J. \& COLWELL, R.K., 2011.-Estimating species richness: 39-54 (en) MAGURRAN, A.E. \&McGILL, B.J. (eds.) Frontiers in measuring biodiversity. Oxford University Press, New York.

GRANT, B.W., BROWN, K., FERGUSON, G.W., \& GIBBONS, J.W., 1994.- Changes in amphibian biodiversity associated with 25 years of pine forest regeneration: implications for biodiversity management: 355367 (en) MAJUMDAR, S.K., BRENNER, F.J., LOVICH, J.E., SCHALLES, J.F. \& MILLER, E.W. (eds.) Biological diversity: problems and challenges. The Pennsylvania Academy of Science, York, PA.

GUAYARA-BARRAGÁN, M.G. \& BERNAL, M.H., 2012.-Fecundidad y fertilidad en once especies de anuros colombianos con diferentes modos reproductivos. Caldasia, 34(2): 483-496.

GUAYASAMIN, J.M. \& TRUEB, L., 2007.- A new species of Glassfrog (Anura: Centrolenidae) from the lowlands of northwesternEcuador, with comments on centrolenid osteology. Zootaxa, 27-45.

GUAYASAMÍN, J.M., CASTROVIEJO-FISHER, S.,TRUEB, L., AYARZAGÜENA,J.,RADA,M.,\&VILÀ, C., 2009.- Phylogenetic systematics of Glassfrogs (Amphibia: Centrolenidae) and their sister taxon Allophryneruthveni. Zootaxa, 2100: 1-97.

HADDAD, C.F.B. \& SAWAYA, R.J., 2000.-Reproductive modes of Atlantic forest Hylid frogs: a general overview and the description of a new mode. Biotropica, 32 (4b): 862-871.DOI: 10.1111/j.17447429.2000.tb00624.x.

HAMMER, Ø., HARPER, D.A.T. \& PAUL, D.R., 2001.-PAST: Paleontological Statistics: analysis software package for education and data. Palaeontología Electrónica, 4 (1): art. 4. Disponible en: http://palaeo- 
electronica.org/2001_1/past/issue1_01.htm

HAMMER, O., HARPER, D.A.T. \& RYAN. P.D., 2005.-PAST, Palaeontological Statistics. Version 1.37.

HEATWOLE, H., \& WILKINSON, J.W., 2012.- Decline and conservation of amphibians: an update. Frog Log, 100:66-68.

HELTSHE, J. \& FORRESTER, N.E., 1983.- Estimating species richness using the jackknife procedure. Biometrics, 39: 1-11.DOI: 10.2307/2530802.

HERRERA-M., A., OLAYA-M., L.A., \& CASTRO-H., F., 2004.-Incidencia de la perturbación antrópica en la diversidad, la riqueza y la distribución de Eleutherodactylus (Anura: Leptodactylidae) en un bosque nublado del suroccidente colombiano. Caldasia, 26(1): 265-274.

HEYER, W.R., DONNELLY, M.A., McDIARMID, R.W., HAYEK, A.C. \& FOSTER, M.S., 1994.-Medición y monitoreo de la diversidad biológica. Métodos estandarizados para anfibios. Smithsonian Institution Press, Washington y Londres, Editorial Universitaria de la Patagonia.

HUEY, R.B., 1991.- Physiological consequences of habitat selection. American Naturalist, 137: 91-115.

INGARAMO, M.D.R., ETCHEPARE, E.G., ÁLVAREZ, B.B. \& PORCEL, E., 2012.- Riqueza y composición de la fauna de anuros en la región oriental de la Reserva Natural Provincial Esteros del Iberá, Corrientes, Argentina. Revista de Biología Tropical, 60(2): 759-769.

IUCN., 2014.-The IUCN Red List of Threatened Species. Version 2014.2. Disponible en: http://www. iucnredlist.org

JONGSMA, G.F., HEDLEY, R.W., DURÃES, R., \& KARUBIAN, J., 2014.- Amphibian diversity and species composition in relation to habitat type and alteration in the Mache-Chindul Reserve, Northwest Ecuador. Herpetologica, 70 (1): 34-46. Doi: http://dx.doi.org/10.1655/HERPETOLOGICA-D-12-00068.

KEENELYSIDE, K., DUDLEY, N., CAIRNS, S., HALL, C. \& STOLTON, S., 2012.-Ecological restoration for protected areas: principles, guidelines and best practices. IUCN, Gland, Switzerland.

LYNCH, J.D. \& MYERS, C.W., 1983.-Frogs of the fitzingeri group of Eleutherodactylus in eastern Panama and Chocoan South America (Leptodactylidae). Bulletin of the American Museum of Natural History, 175 (5): 484-568.

LYNCH, J.D., RUIZ, P.M. \& ARDILA-ROBAYO, M.C., 1997.- Biogeographic Patterns of Colombian Frogs and Toads. Revista de la Academia Colombiana de Ciencias, 21(80): 237-248.

LYNCH, J.D. \& SUARÉZ, A.M., 2004.- Catálogo de Anfibios en el Chocó Biogeográfico: 654-667 (en) RANGEL, O. (ed.) Colombia Diversidad Biótica IV. El Chocó biogeográfico / Costa Pacífica. Universidad Nacional de Colombia, Facultad de Ciencias Instituto de Ciencias Naturales, Bogotá.

MARSH, D.M., FEGRAUS, E.H. \& HARRISON, S., 1999.-Effects of breeding pond isolation on the spatial and temporal dynamics of pond use by the Tungara Frog,Physalaemuspustulosus. Journal of Animal Ecology, 68: 804-814.DOI: 10.1046/j.1365-2656.1999.00332.x.

MENDOZA, A.M. \& ARITA, H.T., 2014.- Priority setting by sites and by species using rarity, richness and phylogenetic diversity: the case of neotropicalglassfrogs (Anura: Centrolenidae). Biodiversity and Conservation, 23(4): 909-926. DOI:10.1007/ s10531-014-0642-5.

MONTES, A.H., OLAYA-M., L.A., \& CASTRO-H., F., 2004.-Incidencia de la perturbación antrópica en la diversidad, la riqueza y la distribución de Eleutherodactylus(anura: leptodactylidae) en un bosque nublado del suroccidente colombiano. Caldasia, 26(1): 265-274.

MORENO-VALDERRAMA, H., VARGAS-CASTAÑO, C.A., LÓPEZ-GALLO, M.P., MONTOYA-OLAYA, H.F., ROBLEDO-CASTAÑEDA, C., YEPES-MEDINA, R.A. et al., 2006.-Plan de manejo 2005-2009 Parque Nacional Natural y patrimonio mundial Los Katíos. Unidad Administrativa Especial del Sistema de Parques Nacionales Naturales de Colombia, Territorial Noroccidental, Turbo, Antioquia.

MUÑOZ-GUERRERO, J.,SERRANO, V.H. \& RAMÍREZ-PINILLA, M.P., 2007.-Microhabitat use, diet and time of activity of four sympatric Neotropical hylid frogs (Anura: Hylidae). Caldasia, 29(2): 413-425.

NAVAS, C.A., CARVAJALINO FERNÁNDEZ, J.M., SABOYÁ ACOSTA, L.P., RUEDA SOLANO, L.A. \& CARVAJALINO FERNÁNDEZ, M.A., 2013.-The body temperature of active amphibians along a tropical elevation gradient: patterns of mean and variance and inference from environmental data.Functional Ecology, 27(5); 1145-1154. DOI: 10.1111/1365-2435.12106.

OSORNO, M., 1999.- Evaluación del efecto de borde para poblaciones de Eleutherodactylus viejas (Amphibia: Anura: Leptodactylidae), frente acorredores de servidumbre en diferenteestado de regeneración, en dos bosquesintervenidos por líneas de transmisióneléctrica de alta tensión. Revista de la Academia Colombiana de Ciencias Exactas, Físicas y Naturales, Suplemento Especial, 23: 347-356.

PEARMAN, P.B., 1997.- Correlates of amphibian diversity in an altered landscape of Amazonian Ecuador. Conservation Biology, 11:1211-1225.

PIPER, W.H., 2011.- Making habitat selection more "familiar": a review. Behavioral Ecology and Sociobiology, 65(7): 1329-1351. DOI: 10.1007/s00265-011-1195-1.

REZA, M.I.H. \& ABDULLAH, S.A., 2011.- Regional Index of Ecological Integrity: A need for sustainable management of natural resources.Ecological indicators, 11(2): 220-229. DOI: 10.1016/j. ecolind.2010.08.010.

RUEDA-ALMONACID, J.V., LYNCH, J.D. \& AMÉZQUITA, A., 2004.-Libro rojo de los Anfibios de Colombia. Serie Libro rojos de especies amenazadas de Colombia. ICN-Universidad Nacional de Colombia, Ministerio del Medio Ambiente, Conservación Internacional Colombia, Bogotá.

STORFER, A., 2003.- Amphibian declines: future directions. Diversity and Distributions, 9: 151-163.DOI: 10.1046/j.1472-4642.2003.00014.x. 
URBINA-C., J.N. \& LONDOÑO-M., M.C., 2003.-Distribución de la comunidad de herpetofauna asociada a cuatro áreas con diferente grado de perturbación en la Isla Gorgona, Pacífico colombiano. Revista de la Academia Colombiana de Ciencias Exactas, Físicas y Naturales, 27(102): 105-113.

URBINA-C., J.N., OLIVARES-P., M. \& REYNOSO, V.H., 2006.-Herpetofauna diversity and microenvironment correlates across a pasture-edge-interior ecotone in tropical rainforest fragments in the Los Tuxtlas Biosphere Reserve of Veracruz, Mexico. Biological Conservation, 132: 61-75. DOI: 10.1016/j. biocon.2006.03.014.

VARGAS, F. \& BOLAÑOS, M., 1999.- Anfibios y reptiles presentes en hábitats perturbados de selva lluviosa tropical enel bajo Anchicayá, Pacílcocolombiano. Revista Colombiana de Ciencias Exactas, Físicas y Naturales, Suplemento Especial, 23: 499-508.

VARGAS, F. \& CASTRO-H., F., 1999.-Distribución y preferencias de micro-hábitat en anuros (Amphibia) en bosque maduro y áreas perturbadas en Anchicayá, Pacífico colombiano.Caldasia, 21: 95-109.

WAKE, D.B. \& VREDENBURG. V.T., 2008.-Are we in the midst of the sixth mass extinction? A view from the world of amphibians. Proceedings of the National Academy of Sciences of the United States of America, 105:11466-11473.

WELLS, K. \& SCHWARTZ, J., 1982.- The effect of vegetation on the propagation of calls in the neotropical frog Centrolenellafleischmanni. Herpetologica, 38 (4): 449-455.

XIMENEZ, S.S., GONÇALVEZ, T.P., \& OLIVEIRA, M.C., 2012.-Substrate color selection by tadpoles of Physalaemus gracilis (Boulenger, 1883)(Anura, Leiuperidae). Pan-American Journal of Aquatic Sciences, 7(2): 111-116. 